\title{
Effects of Jet Quenching on the Hydrodynamical Evolution of Quark-Gluon Plasma
}

\author{
A. K. Chaudhuri ${ }^{1, *}$ and Ulrich Heinz ${ }^{2}$ \\ ${ }^{1}$ Variable Energy Cyclotron Centre, 1-AF, Bidhan Nagar, Kolkata-700 064, India \\ ${ }^{2}$ Department of Physics, The Ohio State University, Columbus, Ohio 43210, USA
}

(Received 10 November 2005; revised manuscript received 12 April 2006; published 9 August 2006)

We study the effects of jet quenching on the hydrodynamical evolution of the quark-gluon plasma (QGP) fluid created in a heavy-ion collision. In jet quenching, a hard QCD parton, before fragmenting into a jet of hadrons, deposits a fraction of its energy in the medium, leading to suppressed production of high- $p_{T}$ hadrons. Assuming that the deposited energy quickly thermalizes, we simulate the subsequent hydrodynamic evolution of the QGP fluid. For partons moving at supersonic speed, $v_{p}>c_{s}$, and sufficiently large energy loss, a shock wave forms leading to conical flow. The PHENIX Collaboration recently suggested that observed structures in the azimuthal angle distribution might be caused by conical flow. We show here that, for phenomenologically acceptable values of parton energy loss, conical flow effects are too weak to explain these structures.

DOI: 10.1103/PhysRevLett.97.062301

PACS numbers: 25.75.- $\mathrm{q}, 13.85 . \mathrm{Hd}, 13.87 .-\mathrm{a}$

Recent $\mathrm{Au}+\mathrm{Au}$ collision experiments at the Relativistic Heavy-Ion Collider (RHIC) saw a dramatic suppression of hadrons with high transverse momenta ("high- $p_{T}$ suppression") [1], and the quenching of jets in the direction opposite to a high- $p_{T}$ trigger particle [2,3], when compared with $p+p$ and $d+$ Au collisions. This is taken as evidence for the creation of a very dense, color opaque medium of deconfined quarks and gluons [4]. Independent evidence for the creation of dense, thermalized quark-gluon matter, yielding comparable estimates for its initial energy density $\left(\langle e\rangle \geqslant 10 \mathrm{GeV} / \mathrm{fm}^{3}\right.$ at time $\tau_{\text {therm }} \lesssim 0.6 \mathrm{fm} / \mathrm{c}$ [5]), comes from the observation of strong elliptic flow in noncentral $\mathrm{Au}+\mathrm{Au}$ collisions [1], consistent with ideal fluid dynamical behavior of the bulk of the matter produced in these collisions.

These two observations raise the question of what happens - in the small fraction of collision events where a hard scattering produces a pair of high- $p_{T}$ partons - to the energy lost by the parton traveling through the medium. The STAR Collaboration has shown that, while in central $\mathrm{Au}+\mathrm{Au}$ collisions there are no hard particles left in the direction opposite to a high- $p_{T}$ trigger particle, one sees enhanced production (compared to $p+p$ ) of soft (low- $p_{T}$ ) particles, broadly distributed over the hemisphere diametrically opposite to the trigger particle [6]. This shows that the energy of the fast parton originally emitted in the direction opposite to the trigger particle is not lost, but severely degraded by interactions with the medium. As the impact parameter of the collisions decreases, the average momentum of the particles emitted opposite to the trigger particle approaches the mean value associated with all soft hadrons, i.e., the $\left\langle p_{T}\right\rangle$ of the thermalized medium [6]. This suggests that the energy lost by the fast parton has been largely thermalized. Nevertheless, this energy is deposited locally along the fast parton's trajectory, leading to local energy density inhomogeneities which, if thermalized, should in turn evolve hydrodynamically. This would modify the usual hydrodynamic expansion of the collision fireball as observed in the overwhelming number of soft collision events where no high- $p_{T}$ partons are created.

Since the fast parton moves at supersonic speed, it was suggested in Ref. [7] that a Mach shock ("sonic boom") should develop, resulting in conical flow and preferred particle emission at a specific angle away from the direction of the fast parton which lost its energy. This Mach angle is sensitive to the medium's speed of sound, $c_{s}$, and thus offers the possibility to measure one of its key properties. A recent analysis by the PHENIX Collaboration [8] of azimuthal dihadron correlations in $200 \mathrm{~A} \mathrm{GeV} \mathrm{Au}+\mathrm{Au}$ collisions revealed structures in the angular distribution which might be suggestive of conical flow.

The idea of Mach shock waves traveling through compressed nuclear matter was first advocated 30 years ago $[9,10]$, but RHIC collisions for the first time exhibit [5] the kind of ideal fluid behavior which might make an extraction of the speed of sound conceivable. An alternate scenario, in which the color wake field generated by the fast colored parton traveling through a quark-gluon plasma accelerates soft colored plasma particles in the direction perpendicular to the wake front $[11,12]$, leads to an emission pattern which is sensitive to the propagation of plasma rather than sound waves [12]. In a strongly coupled plasma with overdamped plasma oscillations, which seems to be the preferred interpretation of RHIC data [13,14], the wake field scenario should reduce to the hydrodynamic Mach cone picture. We here study the dynamical consequences of the latter, going beyond the discussion of linearized hydrodynamic equations in a static background offered in [7].

We assume that just before hydrodynamics become applicable, a pair of high- $p_{T}$ partons is produced near the surface of the fireball. One of them moves outward and escapes, forming the trigger jet, while the other enters into the fireball along, say, the $-x$ direction. The fireball is expanding and cooling. The ingoing parton travels at the speed of light and loses energy in the fireball, which 
thermalizes and acts as a source of energy and momentum for the fireball medium. We model this medium as an ideal fluid with vanishing net baryon density. Its dynamics is controlled by the energy-momentum conservation equations

$$
\partial_{\mu} T^{\mu \nu}=J^{\nu},
$$

where the energy-momentum tensor has the ideal fluid form $T^{\mu \nu}=(\varepsilon+p) u^{\mu} u^{\nu}-p g^{\mu \nu}$, with energy density $\varepsilon$ and pressure $p$ being related by the equation of state (EOS) $p=p(\varepsilon), u^{\mu}=\gamma\left(1, v_{x}, v_{y}, 0\right)$ is the fluid 4-velocity, and the source current $J^{\nu}$ is given by

$$
\begin{gathered}
J^{\nu}(x)=J(x)(1,-1,0,0), \\
J(x)=\frac{d E}{d x}(x)\left|\frac{d x_{\text {jet }}}{d t}\right| \delta^{3}\left(\boldsymbol{r}-\boldsymbol{r}_{\text {jet }}(t)\right) .
\end{gathered}
$$

Massless partons have lightlike 4-momentum, so the current $J^{\nu}$ describing the 4-momentum lost and deposited in the medium by the fast parton is taken to be lightlike, too. $\boldsymbol{r}_{\text {jet }}(t)$ is the trajectory of the jet moving with speed $\left|d x_{\text {jet }} / d t\right|=c \cdot \frac{d E}{d x}(x)$ is the energy loss rate of the parton as it moves through the liquid. It depends on the fluid's local rest frame particle density. Taking guidance from the phenomenological analysis of parton energy loss observed in $\mathrm{Au}+\mathrm{Au}$ collisions at RHIC [15] we take

$$
\frac{d E}{d x}=\left.\frac{s(x)}{s_{0}} \frac{d E}{d x}\right|_{0}
$$

where $s(x)$ is the local entropy density without the jet. The measured suppression of high- $p_{T}$ particle production in $\mathrm{Au}+\mathrm{Au}$ collisions at RHIC was shown to be consistent with a parton energy loss of $\left.\frac{d E}{d x}\right|_{0}=14 \mathrm{GeV} / \mathrm{fm}$ at a reference entropy density of $s_{0}=140 \mathrm{fm}^{-3}$ [15]. For comparison, we also perform simulations with 10 times larger energy loss, $\left.\frac{d E}{d x}\right|_{0}=140 \mathrm{GeV} / \mathrm{fm}$.

For the hydrodynamic evolution we use AZHYDRO $[5,16]$, the only publicly available relativistic hydrodynamic code for anisotropic transverse expansion. This algorithm is formulated in $(\tau, x, y, \eta)$ coordinates, where $\tau=\sqrt{t^{2}-z^{2}}$ is the longitudinal proper time, $\eta=\frac{1}{2} \ln \left[\frac{t+z}{t-z}\right]$ is space-time rapidity, and $\boldsymbol{r}_{\perp}=(x, y)$ defines the plane transverse to the beam direction $z$. AZHYDRO employs longitudinal boost invariance along $z$ but this is violated by the source term (3). We therefore modify the latter by replacing the $\delta$-function in (3) by

$$
\begin{aligned}
\delta^{3}\left[\boldsymbol{r}-\boldsymbol{r}_{\mathrm{jet}}(t)\right] & \rightarrow \frac{1}{\tau} \delta\left[x-x_{\mathrm{jet}}(\tau)\right] \delta\left[y-y_{\mathrm{jet}}(\tau)\right] \\
& \rightarrow \frac{1}{\tau} \frac{e^{-\left[\boldsymbol{r}_{\perp}-\boldsymbol{r}_{\perp, \mathrm{jet}}(\tau)\right]^{2} /\left(2 \sigma^{2}\right)}}{2 \pi \sigma^{2}}
\end{aligned}
$$

with $\sigma=0.35 \mathrm{fm}$. Intuitively, this replaces the "needle" (jet) pushing through the medium at one point by a "knife" cutting the medium along its entire length along the beam direction. The resulting "wedge flow" is ex- pected to leave a stronger signal in the azimuthal particle distribution $d N / d \phi$ than "conical flow" induced by a single parton, since in the latter case one performs an implicit $\phi$-average when summing over all directions of the cone normal vector. While a complete study of this would require a full $(3+1)$-dimensional hydrodynamic calculation, the present boost-invariant simulation should give a robust upper limit for the expected angular signatures. We show that the angular structures predicted from wedge flow are too weak to explain the experimentally observed $\phi$-modulation [8].

The modified hydrodynamic equations in $(\tau, x, y, \eta)$ coordinates read [16]

$$
\begin{gathered}
\partial_{\tau} \tilde{T}^{\tau \tau}+\partial_{x}\left(\tilde{v}_{x} \tilde{T}^{\tau \tau}\right)+\partial_{y}\left(\tilde{v}_{y} \tilde{T}^{\tau \tau}\right)=-p+\tilde{J}, \\
\partial_{\tau} \tilde{T}^{\tau x}+\partial_{x}\left(v_{x} \tilde{T}^{\tau x}\right)+\partial_{y}\left(v_{y} \tilde{T}^{\tau x}\right)=-\partial_{x} \tilde{p}-\tilde{J},
\end{gathered}
$$
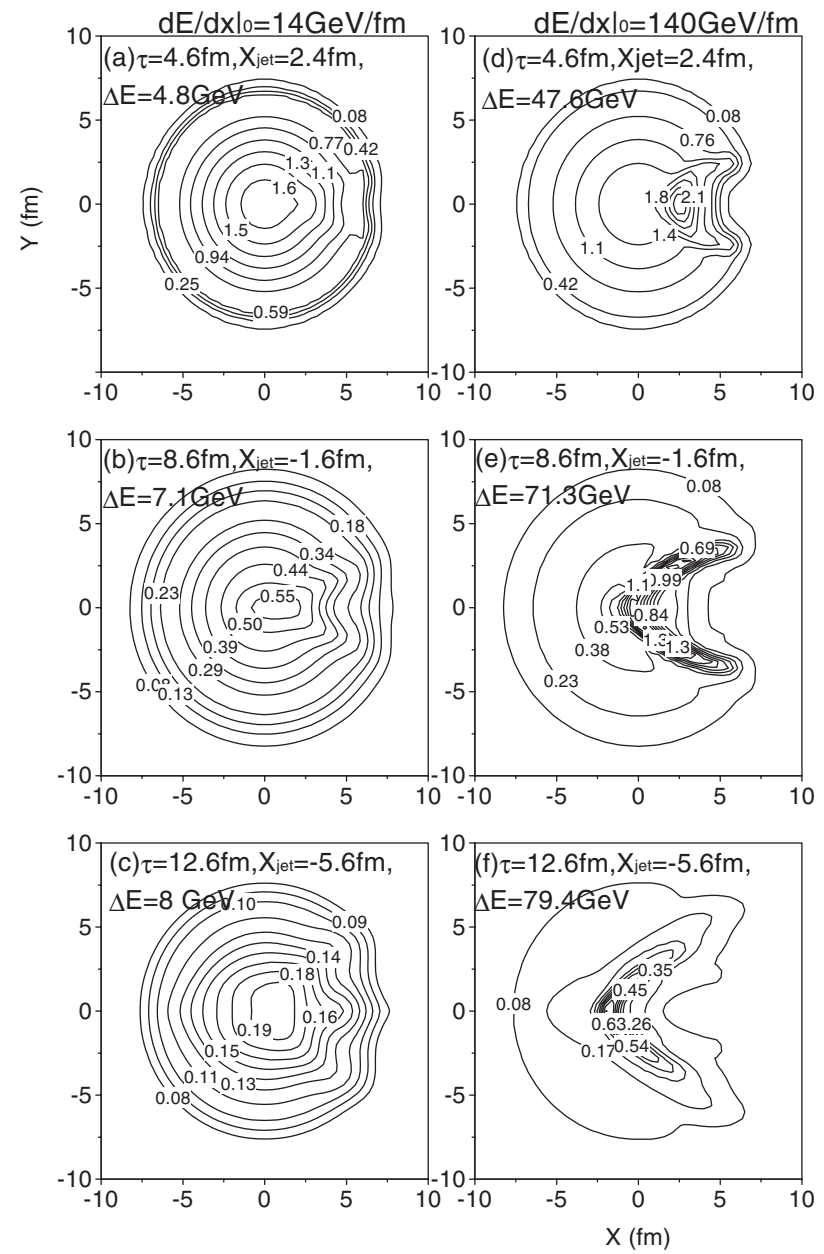

FIG. 1. Contours of constant local energy density in the $x-y$ plane at three different times, $\tau=4.6,8.6$, and $12.6 \mathrm{fm} / c$. In each case the position of the fast parton, along with the integrated energy loss $\Delta E=\int J(x) d x d y d \tau$ up to this point, is indicated at the top of the figure. Diagrams (a)-(c) in the left column were calculated with a reference energy loss $d E /\left.d x\right|_{0}=$ $14 \mathrm{GeV} / \mathrm{fm}$, those in the right column [panels (d)-(f)] with a 10 times larger value. 


$$
\partial_{\tau} \tilde{T}^{\tau y}+\partial_{x}\left(v_{x} \tilde{T}^{\tau y}\right)+\partial_{y}\left(v_{y} \tilde{T}^{\tau y}\right)=-\partial_{y} \tilde{p},
$$

where $\tilde{T}^{\mu \nu}=\tau T^{\mu \nu}, \tilde{v}_{i}=T^{\tau i} / T^{\tau \tau}, \tilde{p}=\tau p$, and $\tilde{J}=\tau J$.

To simulate central Au + Au collisions at RHIC, we use the standard initialization described in [5] and provided in the downloaded AZHYDRO input file [16], corresponding to a peak initial energy density of $\varepsilon_{0}=30 \mathrm{GeV} / \mathrm{fm}^{3}$ at $\tau_{0}=$ $0.6 \mathrm{fm} / c$. We use the equation of state EOS-Q described in $[5,16]$ incorporating a first order phase transition and hadronic chemical freeze-out at a critical temperature $T_{c}=$ $164 \mathrm{MeV}$. The hadronic sector of EOS-Q is soft with a squared speed of sound $c_{s}^{2} \approx 0.15$.

In our study the quenching jet starts from $x_{\text {jet }}=6.4 \mathrm{fm}$ at $\tau_{0}=0.6 \mathrm{fm}$, moving left towards the center with constant speed $v_{\text {jet }}=c$. For an upper limit on conical flow effects, the fast parton is assumed to have sufficient initial energy to emerge on the other side of the fireball. To simulate cases where the fast parton has insufficient energy to fully traverse the medium we have also done simulations where the parton loses all its energy within an (arbitrarily chosen) distance of $6.4 \mathrm{fm}$. We further compared with a run
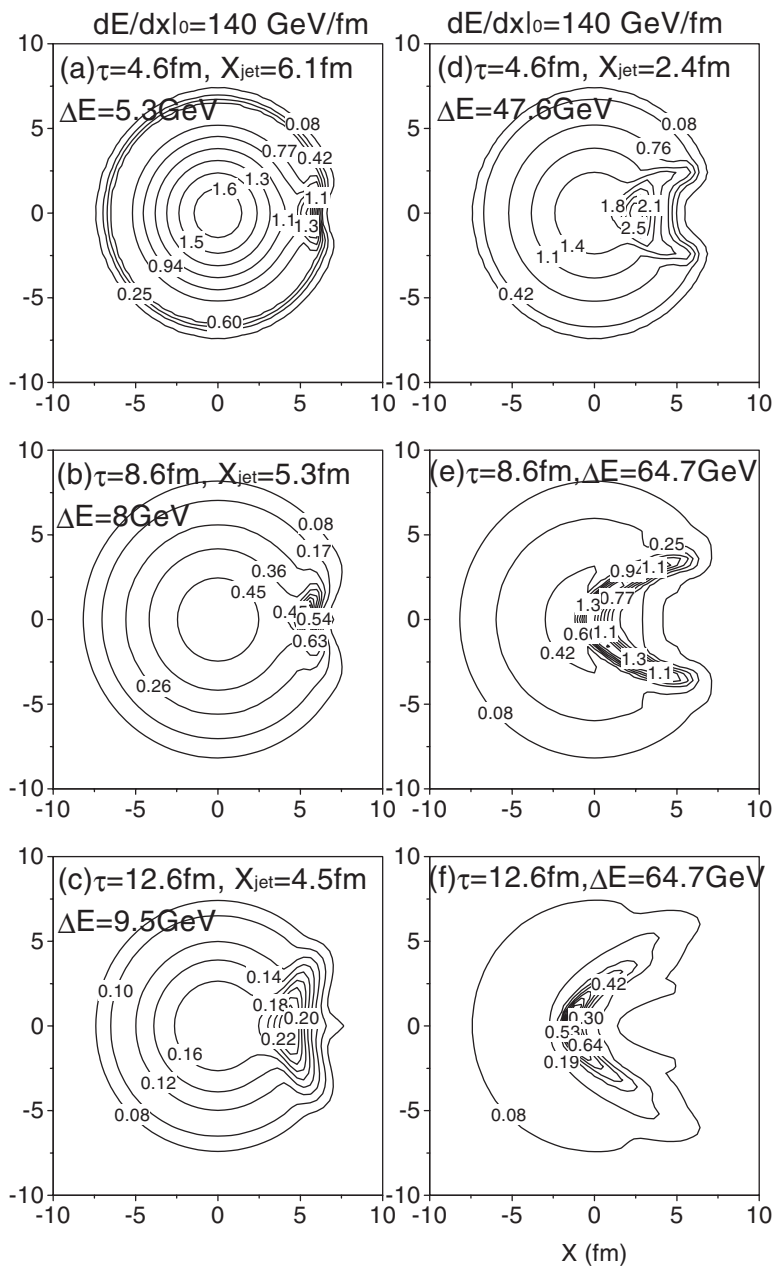

FIG. 2. As in Fig. 1 but for a parton moving at subsonic speed $v_{\text {jet }}=0.2 c$ (left column) and for a fast parton $\left(v_{\text {jet }}=c\right.$ ) which loses all its energy within the first $6.4 \mathrm{fm}$ (right column). where the parton moved at (constant) subsonic speed $\left(v_{\text {jet }}=0.2 c<c_{s}\right)$.

The resulting evolution of the energy density of the QGP fluid is shown in Figs. 1 and 2. The left column of Fig. 1 shows results for a phenomenologically acceptable value $\left.\frac{d E}{d x}\right|_{0}=14 \mathrm{GeV} / \mathrm{fm}$ [15] for the reference parton energy loss whereas in all other columns we use a 10 times larger energy loss. The width of the Gaussian source [see Eq. (5)] is $\sigma=0.7 \mathrm{fm}$. In the left column of Fig. 1 the effects of the energy deposition from the fast parton are hardly visible. Only for a much larger energy loss (right column) we recognize a clear conical flow pattern. The accumulating wave fronts from the expanding energy density waves build up a sonic boom shock front which creates a Mach cone. The right columns in Figs. 1 and 2 show that the cone normal vector forms an angle $\theta_{M}$ with the direction of the quenching jet that is qualitatively consistent with expectations from the theoretical relation $\cos \theta_{M}=c_{s} / v_{\text {jet }}$. However, this angle is not sharply defined since the cone surface curves due to inhomogeneity and radial expansion of the underlying medium. This differs from the static homogeneous case [7].

When the parton travels at a subsonic speed $v_{\text {jet }}=$ $0.2 c<c_{s}$ (left column in Fig. 2), it does not get very far before the fireball freezes out due to longitudinal expansion. In this case one only observes an accumulation of energy around the parton but no evidence of Mach cone formation. When the parton travels with $v_{\text {jet }}=c$ but looses all its energy after $6.4 \mathrm{fm}$ before fully traversing the fireball (right column in Fig. 2), the fireball evolution beyond $\tau=$ $7 \mathrm{fm}$ is not affected by the fast parton directly but only indirectly through the propagation of earlier deposited energy. Still, Figs. 1(f) and 2(f) show that the late time evolution of the fireball is quite similar in both cases, demonstrating that energy deposition by the fast parton during the late fireball stages is small, due to dilution of the matter, and can almost be neglected.

Tests with different values for the width $\sigma$ of the Gaussian profile in Eq. (5) for the deposited energy show that the cone angle gets better defined for smaller source size $\sigma$. Note that the quenching jet destroys the azimuthal symmetry of the initial energy density distribution but leaves the azimuthally symmetric energy contours to the left of the jet unaffected.

We close with a discussion of observable consequences of conical flow. One expects [7] azimuthally anisotropic particle emission, peaking at angles $\phi=\pi \pm \theta_{M}$ relative to the trigger jet where $\theta_{M}$ is the Mach angle. Using the standard Cooper-Frye prescription, we have computed the angular distribution of directly emitted pions at a freezeout temperature $T_{\mathrm{fo}}=100 \mathrm{MeV}$ [5].

Figure 3 shows the azimuthal distribution of $\pi^{-}$from a variety of different simulations. [The $\phi$-independent constant $\left(d N_{\pi^{-}} / d y d \phi\right)_{\text {no jet }}=27$ from central collisions without jets has been subtracted.] For $d E /\left.d x\right|_{0}=14 \mathrm{GeV} / \mathrm{fm}$ the azimuthal modulation is very small. In none of the 


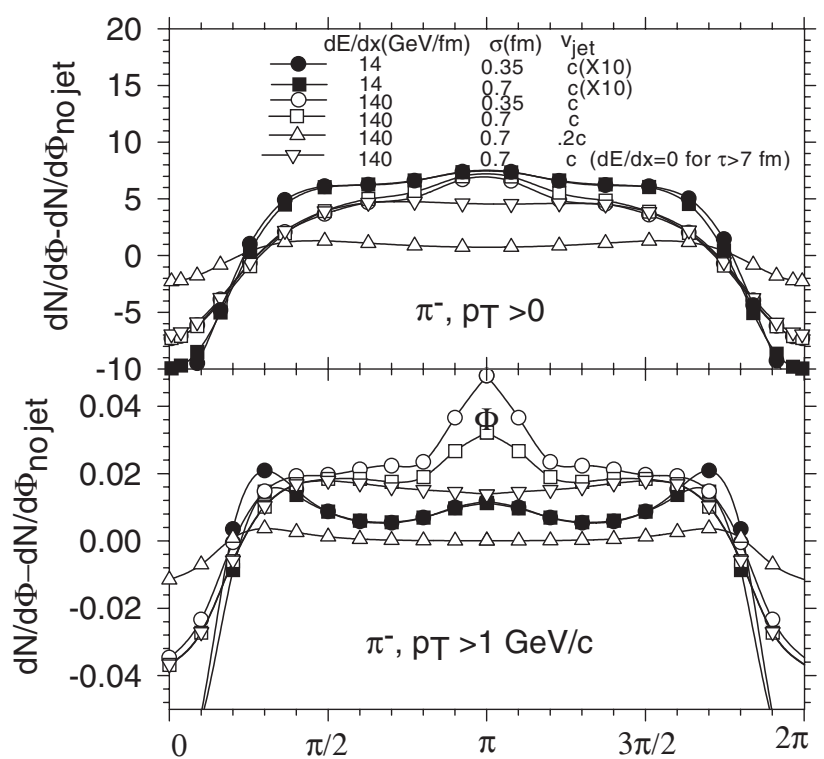

FIG. 3. Azimuthal distribution $d N / d y d \phi$ of negative pions per unit rapidity. In the upper panel we integrate over all $p_{T}$ while the lower panel shows only pions with $p_{T}>1 \mathrm{GeV} / c$. Different symbols refer to different parameters as indicated. For better visibility the $\phi$-independent rate in the absence of the quenching jet has been subtracted. Filled symbols show the realistic case $d E /\left.d x\right|_{0}=14 \mathrm{GeV} / \mathrm{fm}$, enhanced by a factor of 10 for visibility.

cases studied we find peaks at the predicted Mach angle with an associated dip in the direction of the quenched jet at $\phi=\pi$. One rather sees a peak at $\phi=\pi$, broadened by shoulders on both sides which turn into small peaks at (relative to the quenching jet) backward angles if thermal smearing is reduced by considering only high- $p_{T}$ pions. The peak at $\phi=\pi$ is absent when the parton loses all its energy halfway through the fireball or is too slow to get to the other side before freeze-out, suggesting that it reflects the directed momentum imparted on the medium by the fast parton. It is slightly more accentuated for smaller $\sigma$ and higher $p_{T}$. We also found that the width of the shoulders is almost independent of the speed of sound of the medium and can not be used to diagnose the stiffness of its equation of state. The shoulders exist even for subsonic parton propagation ( $v_{\text {jet }}=0.2 c$, upright triangles), showing that other mechanisms (such as back splash from the hard parton hitting the fluid and a general bias of the energy deposition towards the right side of the fireball due to the higher density of the medium at early times) have a strong influence on the angular distribution of the emitted particles which interferes with the position of the Mach peaks. The shoulder resulting from this combination of effects is much broader than the angular structures seen in the data [8]. The absence of a clear dip at $\phi=\pi$ in our simulations is all the more troubling since it should have been stronger for the wedge flow studied here than for real "conical" flow.
Our calculation does not average over the initial production points of the trigger particle, i.e., it ignores that in most cases its quenching partner does not travel right through the middle of the fireball cylinder, but traverses it semitangentially. This should further decrease the prominence of the shoulders in $d N / d \phi$. We conclude that conical flow may be able to explain the broadening of the away side peak in the hadron angular correlation function around $\phi=\pi$ pointed out by the STAR Collaboration [6], but is unlikely to be responsible for the relatively sharp structures near $\phi=\pi \pm 1$ seen by PHENIX [8]. This conclusion extends to other conical flow phenomena, such as those generated by color wake fields $[11,12]$.

This work was supported by the U.S. Department of Energy under Contract No. DE-FG02-01ER41190.

*Corresponding author.

Electronic address: akc@ veccal.ernet.in

[1] I. Arsene et al. (BRAHMS Collaboration), Nucl. Phys. A757, 1 (2005); B. B. Back et al. (PHOBOS Collaboration), ibid. A757, 28 (2005); J. Adams et al. (STAR Collaboration), ibid. A757, 102 (2005); K. Adcox et al. (PHENIX Collaboration), ibid. A757, 184 (2005).

[2] C. Adler et al. (STAR Collaboration), Phys. Rev. Lett. 90, 082302 (2003); J. Adams et al. (STAR Collaboration), Phys. Rev. Lett. 91, 072304 (2003); Phys. Rev. Lett. 93, 252301 (2004).

[3] J. Rak et al. (PHENIX Collaboration), J. Phys. G 30, S1309 (2004).

[4] M. Gyulassy, I. Vitev, X.-N. Wang, and B.-W. Zhang, in Quark-Gluon Plasma 3, edited by R. C. Hwa and X.-N. Wang (World Scientific, Singapore, 2004), p. 123.

[5] P. F. Kolb and U. Heinz, in Ref. [4], p. 634.

[6] J. Adams et al. (STAR Collaboration), Phys. Rev. Lett. 95, 152301 (2005).

[7] J. Casalderrey-Solana, E. V. Shuryak, and D. Teaney, J. Phys.: Conf. Ser. 27, 22 (2005).

[8] S.S. Adler et al. (PHENIX Collaboration), nucl-ex/ 0507004.

[9] G. F. Chapline, M. H. Johnson, E. Teller, and M. S. Weiss, Phys. Rev. D 8, 4302 (1973).

[10] W. Scheid, H. Müller, and W. Greiner, Phys. Rev. Lett. 32, 741 (1974).

[11] H. Stöcker, Nucl. Phys. A750, 121 (2005).

[12] J. Ruppert and B. Müller, Phys. Lett. B 618, 123 (2005).

[13] U. Heinz and P. F. Kolb, Nucl. Phys. A702, 269 (2002).

[14] M. Gyulassy and L. D. McLerran, Nucl. Phys. A750, 30 (2005); E. Shuryak, Nucl. Phys. A750, 64 (2005).

[15] X. N. Wang, Phys. Rev. C 70, 031901 (2004) and (private communication).

[16] P. F. Kolb, J. Sollfrank, and U. Heinz, Phys. Rev. C 62, 054909 (2000); P. F. Kolb and R. Rapp, Phys. Rev. C 67, 044903 (2003). The computer code can be downloaded from URL http://nt3.phys.columbia.edu/people/molnard/ OSCAR/. 